\title{
CJD survey offers Britain a glimmer of hope
}

London

British health officials are breathing a sigh of relief - for now. Initial results from a survey to assess the extent of infection with the human form of bovine spongiform encephalopathy (BSE) have drawn a blank.

Analyses of more than 3,000 clinical samples, mostly of appendixes removed from patients in the late 1990s, have failed to reveal any signs of the prion protein responsible for new variant Creutzfeldt-Jakob disease (vCJD). Just a handful of positive results would have suggested an epidemic of tens, or even hundreds, of thousands of cases.

But it's a case of no news rather than good news. A sizeable epidemic cannot be ruled out on the basis of these results alone. Technical limitations of the survey, plus uncertainty about the progression of vCJD in infected people, make it difficult to make predictions.

Researchers at the National CJD Surveillance Unit in Edinburgh and Derriford Hospital in Plymouth are analysing some 18,000 appendix and tonsil samples, using immunohistochemical methods to test for prions.
They hope to gauge the extent of vCJD infection in the British population. But while previous research on tissues from vCJD victims has shown that the prion accumulates in appendixes and tonsils, no one knows how early in the course of infection it appears.

Leszek Borysiewicz of the University of Wales College of Medicine in Cardiff, who chairs the scientific committee overseeing the studies, stresses the limitations of the study. "This sample size is small," he says. The tissue was also preserved with fixative chemicals that could make traces of prion hard to spot.

John Collinge of Imperial College, London, is now conducting a study of some 2,000 freshly collected tonsil samples, and will use a western blot test to detect the vCJD prion. This may yield better information. But by the time these results are available, researchers led by Roy Anderson at the Wellcome Trust Centre for the Epidemiology of Infectious Disease in Oxford claim they may already have defined an upper limit for the eventual size of the vCJD epidemic.

In January, the team published a paper in

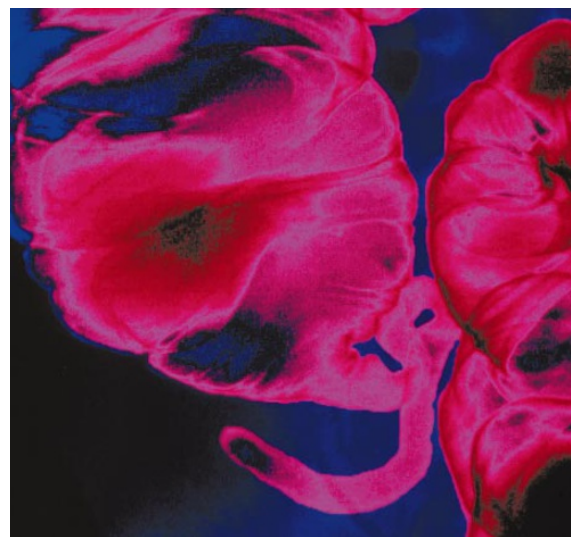

Organ survey: the appendix, at the bottom of this false-colour X-ray, provides a test for vCJD.

Proceedings of the Royal Society $B(267,23$; 2000) predicting that, if the rate at which new cases of vCJD appear remains at its current level until the end of this year, then the upper limit for the epidemic would be 14,000 cases. So far, at least, there are no signs of an increase in the rate of new cases.

Peter Aldhous

\section{Space-station airlock to serve as temporary lab}

\section{Munich}

Forget purpose-built laboratories, all you need to do experiments in space is an airlock. At least, that is where the first experiments on board the International Space Station (ISS) will take place.

Thanks to a deal with the Russian Space Agency, German and Russian physicists will make an early start to research on the space station, long before its main laboratories reach orbit. Using a makeshift lab, the researchers will begin a multimillion dollar investigation into the properties of a complex plasma later this year.

The location will be the bubble-shaped connection chamber between the Russian service module, due to be launched in June, and the Zarya control module,

already in orbit. Once the ISS is fully operational, the chamber will be a busy thoroughfare, and will have to be kept clear. But until then, the

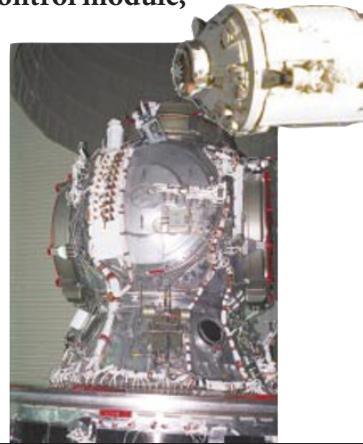

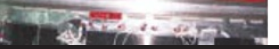

Russian Space Agency is happy to turn the airlock over to the plasma physicists, led by Greg Morfill of the Max Planck Institute for Extraterrestrial Physics in Garching.

Accustomed to a make-do-and-mend approach from their experiences with the ageing Mir station, Russian space officials have even allowed the scientists to drill a

hole through a door hatch in order to thread electricity cables into the airlock. "The agency was very fast and unbureaucratic," says Morfill, who acknowledges that its western counterparts would not have been quite so accommodating. "The same sort of agreement with NASA would have required a square metre of paperwork;

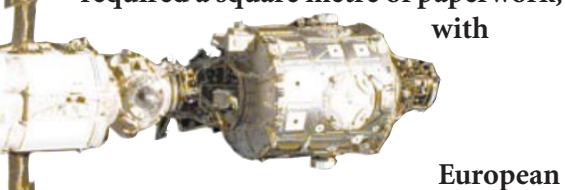

space agencies,

perhaps a square kilometre," he says.

The experiments will examine the unusual plasma states that can be created by introducing microspheres of melanine formaldehyde into normal plasmas. The microspheres, which are around one hundred billion times more massive than the electrons and ions that constitute a plasma, introduce order into what is normally the most disordered form of matter. The plasma liquefies, and then crystallizes. However, gravity disturbs the spheres making it difficult to interpret data from studies of the plasma on Earth.

Morfill and his colleagues had originally hoped to run the experiments on Mir, but when its future started to look shaky, they shifted their allegiance to the ISS. The team will attempt to observe the propagation of waves through the crystals, and will also watch the behaviour of individual microspheres as the crystals melt analagous to studying individual molecules in a conventional crystal. "Because the microspheres are so relatively massive," says Morfill, "we will be able to record their individual movements with [charge-coupled device] cameras."

Russia is paying for the launch costs, the crew and their training, and other logistics, to a value estimated at around DM20 million (US\$9.45 million). Germany is contributing DM8 million.

The first crew to visit the space station in October will tend the apparatus for a week, and bring back 40 hours of videos for Morfill's team to analyse. Based on these results, the experiments will be redesigned and repeated by the third ISS crew a couple of months later. The fifth crew may also continue the experiments. Alison Abbott http://www.mpe.mpg.de/www_th/plasma-crystal/ index.html 